PROCEEDINGS OF THE

AMERICAN MATHEMATICAL SOCIETY

Volume 127, Number 4, April 1999, Pages 1231-1241

S 0002-9939(99)04627-4

\title{
HARMONIC MAPS WITH NONCONTACT BOUNDARY VALUES
}

\author{
HAROLD DONNELLY
}

(Communicated by Peter Li)

\begin{abstract}
Every rank one symmetric space $M$, of noncompact type, admits a compactification $\bar{M}$ by attaching a sphere $S^{n-1}$ at infinity. If $M$ does not have constant sectional curvature, then $\bar{M}-M$ admits a natural contact structure. This paper presents a number of harmonic maps $h$, from $M$ to $M$, which extend continuously to $\bar{M}$, and have noncontact boundary values. If the boundary values are assumed continuously differentiable, then the contact structure must be preserved.
\end{abstract}

\section{INTRODUCTION}

Suppose that $M$ is a complete simply connected manifold of negative curvature. Then $M$ is diffeomorphic to $R^{n}$ and $M$ admits a natural compactification $\bar{M}$, by adding a sphere $S^{n-1}$ at infinity. Given a continuous map $f: S^{n-1} \rightarrow S^{n-1}$, the Dirichlet problem at infinity is to find a harmonic map $h: M \rightarrow M$ which continuously assumes the boundary values $f$ on $S^{n-1}$.

Let $M$ be a rank one symmetric space, other than the hyperbolic space. The sphere $\bar{M}-M$ then admits a generalized contact structure. In [2], it was shown that if $h \in C^{2}(M, M) \cap C^{1}(\bar{M}, \bar{M})$ is harmonic, then its boundary value $f$ is a contact transformation. More generally, if $h$ is only defined in a neighborhood of some boundary point $p \in \bar{M}-M$, then $f$ is locally a contact transformation.

The purpose of the present paper is to show that it is necessary to suppose that the boundary values of $h$ are assumed continuously differentiably in order to guarantee that $f$ is contact, even in a weak sense. In fact, $f$ will be continuously differentiable and noncontact, so $f$ cannot satisfy any reasonable notion of weakly contact. In Proposition 3.5, we give an explicit example of a harmonic map $h \in$ $C^{2}(M, M) \cap C^{0}(\bar{M}-\infty)$ having noncontact boundary values $f$. Here $\infty$ denotes the point at infinity in the unbounded model. Section 4 extends the example of Proposition 3.5 by constructing an infinite family of such solutions. Finally, we show the existence of harmonic maps $h \in C^{2}(M, M) \cap C^{0}(\bar{M}, \bar{M})$ having noncontact boundary values. The existence proof of Section 5 does not provide an explicit solution, as in Proposition 3.5. However, the solution is shown to exist on all of $\bar{M}$, not just $\bar{M}-\infty$.

Received by the editors April 19, 1997 and, in revised form, July 31, 1997.

1991 Mathematics Subject Classification. Primary 58E20.

The author was partially supported by NSF Grant DMS-9622709.

(C)1999 American Mathematical Society 
The techniques employed in this paper are developed from the earlier work of mathematicians who studied the lack of uniqueness in the Dirichlet problem for harmonic maps between hyperbolic spaces $H^{n}$, i.e. locally symmetric spaces of constant curvature -1 . Li and Tam [4] and Wolf [6] constructed a family of proper harmonic maps $h: H^{2} \rightarrow H^{2}$ having boundary value the identity on the circle at infinity. Economakis [3] generalized this result to $H^{n}$. The method of separation of variables is used to reduce the harmonic map equation to an ordinary differential equation. In higher dimensions, $n>2$, the ordinary differential equation is much more difficult to solve.

\section{BOUNDARY VALUES ASSUMED SMOOTHLY}

Let $M$ be a rank one symmetric space of noncompact type. We exclude the case where $M$ has constant negative sectional curvature. The unbounded model of $M$ is $(0, \infty) \times N$, where $N$ is a two term nilpotent group. The Lie algebra $\mathfrak{n}$ of $N$ decomposes as $\mathfrak{n}=\mathfrak{n}_{1} \oplus \mathfrak{n}_{2}$, where $\mathfrak{n}_{2}$ is central in $\mathfrak{n}$ and $\left[\mathfrak{n}_{1}, \mathfrak{n}_{1}\right] \subset \mathfrak{n}_{2}$. The Cayley transform identifies the compactification $\bar{M}$ of $M$ with $[0, \infty) \times N \cup\{\infty\}$, where $\{\infty\}$ denotes the point at infinity.

For the unbounded model, the metric of $M$ may be realized as a doubly warped product

$$
g_{M}=\left(\frac{d y}{y}\right)^{2}+y^{-2} g_{\mathfrak{n}_{1}}+y^{-4} g_{\mathfrak{n}_{2}} .
$$

Here $y \in(0, \infty)$ is the coordinate on the first factor of $R^{+} \times N$. In this paper, we use indices $0 \leq j \leq n_{1}+n_{2}$. The index 0 refers to $\partial / \partial y$, the indices $1 \leq j \leq n_{1}$ refer to the $g_{\mathfrak{n}_{1}}$ part of $(2.1)$, and the indices $n_{1}+1 \leq j \leq n_{1}+n_{2}$ refer to the $g_{\mathfrak{n}_{2}}$ part of (2.1).

Suppose that $h: M \rightarrow M$ is a $C^{2}$ map. The differential $d h$ is a section of $T^{*} M \otimes h^{-1} T M$. The Levi-Civita connection of $M$ induces a natural connection $\nabla$ on $T^{*} M \otimes h^{-1} T M$. So one has

$$
\nabla d h \in \Gamma\left(T^{*} M \otimes T^{*} M \otimes h^{-1} T M\right) .
$$

The tension field $\tau(h)=\operatorname{Tr}(\nabla d h) \in \Gamma\left(h^{-1} T M\right)$ is obtained by taking the trace in the first two factors. We say that $h$ is harmonic when its tension field vanishes identically.

We will need an explicit formula for $\tau(h)$ in the special case of the metric given by (2.1). Choose an orthonormal basis $X_{1}, X_{2}, \ldots, X_{n_{1}}$ for $\mathfrak{n}_{1}$ and an orthonormal basis $Z_{1}, Z_{2}, \ldots, Z_{n_{2}}$ for $\mathfrak{n}_{2}$, relative to the left invariant metric on $N$. One has

$$
\left[X_{1}, Z_{j}\right]=\left[Z_{i}, Z_{j}\right]=0 \quad \text { and } \quad\left[X_{i}, X_{j}\right]=\sum_{k} a_{i j}^{k} Z_{k}
$$

for some structure constants $a_{i j}^{k}$. We choose a frame field $e_{i}$ on $M$ to consist of $e_{0}=\partial / \partial y, e_{i}=X_{i}, 1 \leq i \leq n_{1}$, and $e_{i}=Z_{i-n_{1}}, n_{1}+1 \leq i \leq n_{1}+n_{2}$. Of course, $e_{i}$ is orthogonal but not orthonormal relative to the metric given by (2.1). If $e_{i}^{*}$ is the dual coframe field, then the differential of $h$ has components $d h=\sum_{i, j} h_{i}^{j} e_{i}^{*} \otimes e_{j}$. Similarly, the metric on the cotangent bundle has components $g^{i j}=\left\langle e_{i}^{*}, e_{j}^{*}\right\rangle$. We denote $h_{i j}^{k}=e_{j} h_{i}^{k}$ to be the derivatives of the $h_{i}^{k}$ in the direction $e_{j}$. 
The tension field $\tau(h)$ is a section of $h^{-1} T M$. We denote the normal variable on the image by $\bar{y}=y \circ h$. In [2], the components of $\tau(h)$ were calculated explicitly as

$$
\begin{aligned}
\tau^{0}(h)= & g^{j j} h_{j j}^{0}+\left(1-n_{1}-2 n_{2}\right) h_{0}^{0} y-g^{j j} h_{j}^{0} h_{j}^{0} \bar{y}^{-1} \\
& +g^{j j} \sum_{\gamma=1}^{n_{1}} h_{j}^{\gamma} h_{j}^{\gamma} \bar{y}^{-1}+g^{j j} \sum_{\gamma=n_{1}+1}^{n_{1}+n_{2}} 2 h_{j}^{\gamma} h_{j}^{\gamma} \bar{y}^{-3}, \\
\tau^{\alpha}(h)= & g^{j j} h_{j j}^{\alpha}+\left(1-n_{1}-2 n_{2}\right) h_{0}^{\alpha} y-2 g^{j j} h_{j}^{0} h_{j}^{\alpha} \bar{y}^{-1} \\
& +g^{j j} \sum_{\beta=1}^{n_{1}} \sum_{\gamma=n_{1}+1}^{n_{1}+n_{2}} a_{\alpha \beta}^{\gamma-n_{1}} h_{j}^{\beta} h_{j}^{\gamma} \bar{y}^{-2}, \quad 1 \leq \alpha \leq n_{1}, \\
\tau^{\alpha}(h)= & g^{j j} h_{j j}^{\alpha}+\left(1-n_{1}-2 n_{2}\right) h_{0}^{\alpha} y-4 g^{j j} h_{j}^{0} h_{j}^{\alpha} \bar{y}^{-1}, n_{1}+1 \leq \alpha \leq n_{1}+n_{2} .
\end{aligned}
$$

Here $j$ is summed from 0 to $n_{1}+n_{2}$. Observe that $\operatorname{dim} M=n_{1}+n_{2}+1$.

Suppose that $U$ is a neighborhood of some boundary point $p \in 0 \times N$. Let $h: U \cap M \rightarrow M$ be a harmonic map having boundary values $f: U \cap(0 \times N) \rightarrow 0 \times N$. We say that $f$ is a contact transformation if it is continuously differentiable and $d f$ satisfies $f_{j}^{\gamma}=0,1 \leq j \leq n_{1}, n_{1}+1 \leq \gamma \leq n_{1}+n_{2}$. Equivalently, $d f\left(\mathfrak{n}_{1}\right) \subset \mathfrak{n}_{1}$. The following result was derived in [2].

Theorem 2.3. Suppose that $h \in C^{2}(U \cap M, M) \cap C^{1}(U \cap \bar{M}, \bar{M})$ is a harmonic map with boundary values $f \in C^{1}(U \cap(0 \times N), 0 \times N)$. Then $f$ is a contact transformation.

For the convenience of the reader, we sketch the proof of Theorem 2.3. Assume that $h \in C^{2}(U \cap \bar{M}, \bar{M})$. The result then follows by multiplying formula (2.2) for $\tau^{0}(h)$ by $\bar{y}^{3} / y^{2}$ and letting $y \rightarrow 0$. Under the weaker hypothesis that $h \in$ $C^{1}(U \cap \bar{M}, \bar{M})$, more work is required. One uses the divergence theorem on small balls and passage to a subsequence to handle the second order terms in (2.2). Full details of the proof are given in [2].

\section{NONLINEAR ORDINARY DIFFERENTIAL EQUATIONS}

In general, it seems formidable to construct solutions to the harmonic map equation $\tau(h)=0$, where $\tau(h)$ is given by (2.2). However, if the boundary value $f: N \rightarrow N$ is a Lie group homomorphism, then the problem simplifies considerably. After imposing an additional algebraic condition, which appears as (3.2) below, one is reduced to solving a nonlinear ordinary differential equation.

Suppose that $f: N \rightarrow N$ is a differentiable map. Let $Y_{i}$ be a left invariant frame field on $T N$. We may write $d f\left(Y_{i}(x)\right)=\sum_{j} b_{i j}(x) Y_{j}(f(x))$, for each $x \in N$. Here $Y_{i}(x)$ belongs to the tangent space $T_{x} N$ at $x$. We say that the components of $d f$ are constant in the left invariant frame field $Y_{i}$ if $b_{i j}(x)=b_{i j}(e)$, for all $x \in N$, where $e$ is the identity element of the group $N$. It is easy to verify that if the components of $d f$ are constant in some left invariant frame field, then the components of $d f$ are constant in any left invariant frame field. The following elementary observation is very useful:

Proposition 3.1. Suppose that $f: N \rightarrow N$ is a Lie group homomorphism. Then the components of $d f$ are constant in any left invariant frame field. 
Proof. Let $f: N \rightarrow N$ be any differentiable map. One has $Y_{i}(x)=x_{*} Y_{i}(e)$, where $x_{*}$ is the differential of left translation by $x$. Therefore

$$
\begin{aligned}
d f\left(x_{*} Y_{i}(e)\right) & =\sum_{j} b_{i j}(x)(f(x))_{*} Y_{j}(e), \\
d f\left(Y_{i}(e)\right) & =\sum_{j} b_{i j}(e)(f(e))_{*} Y_{j}(e) .
\end{aligned}
$$

The condition $b_{i j}(x)=b_{i j}(e)$ is therefore equivalent to

$$
(f(x))_{*}^{-1} \circ d f \circ x_{*}=(f(e))_{*}^{-1} \circ d f
$$

where each side is an endomorphism of $T_{e} N$.

If $f: N \rightarrow N$ is a Lie group homomorphism, then $f(e)=e$, so the above condition reduces to

$$
d f \circ x_{*}=(f(x))_{*} \circ d f .
$$

The definition of homomorphism is that $f(x y)=f(x) f(y)$. We now replace $y$ by a one parameter group $y=\exp (t Y)$ and differentiate in $t$.

Assume now that $f: N \rightarrow N$ is a Lie group homomorphism. Define a map $h: R^{+} \times N \rightarrow R^{+} \times N$ by setting $h(y, n)=(g(y), f(n))$. The tension field $\tau(h)$ of $h$ is given by the equations (2.2). Suppose that the boundary value of $f$ satisfies the algebraic condition

$$
\sum_{j=1}^{n_{1}+n_{2}} g^{j j} \sum_{\beta=1}^{n_{1}} \sum_{\gamma=n_{1}+1}^{n_{1}+n_{2}} a_{\alpha \beta}^{\gamma-n_{1}} f_{j}^{\beta} f_{j}^{\gamma}=0
$$

for $1 \leq \alpha \leq n_{1}$. Then $h$ is harmonic if and only if $g$ satisfies the following ordinary differential equation:

$$
\begin{aligned}
y^{2} g^{\prime \prime}(y) & +\left(1-n_{1}-2 n_{2}\right) y g^{\prime}(y)-y^{2}\left(g^{\prime}(y)\right)^{2}(g(y))^{-1} \\
& +y^{2}\left[\sum_{j=1}^{n_{1}} \sum_{\gamma=1}^{n_{1}} f_{j}^{\gamma} f_{j}^{\gamma}+y^{2} \sum_{j=n_{1}+1}^{n_{1}+n_{2}} \sum_{\gamma=1}^{n_{1}} f_{j}^{\gamma} f_{j}^{\gamma}\right](g(y))^{-1} \\
& +2 y^{2}\left[\sum_{j=1}^{n_{1}} \sum_{\gamma=n_{1}+1}^{n_{1}+n_{2}} f_{j}^{\gamma} f_{j}^{\gamma}+y^{2} \sum_{j=n_{1}+1}^{n_{1}+n_{2}} \sum_{\gamma=n_{1}+1}^{n_{1}+n_{2}} f_{j}^{\gamma} f_{j}^{\gamma}\right](g(y))^{-3}=0 .
\end{aligned}
$$

Recall that the $f_{j}^{\gamma}$ are constant by Proposition 3.1. In order for $h$ to solve the Dirichlet problem, with boundary value $f: N \rightarrow N$, we must impose the boundary condition $g(0)=0$.

If $Z$ denotes the center of $N$, then $N / Z$ is abelian. By choosing $f$ to be a suitable composition of homomorphisms $N \rightarrow N / Z \rightarrow N$, we achieve $f_{1}^{n_{1}+1}=1$ and all other $f_{j}^{\alpha}=0$, for any given $N$. In this special case, equation (3.3) reduces to

$$
g^{3}(y) g^{\prime \prime}(y)-\frac{m}{y} g^{3}(y) g^{\prime}(y)-g^{2}(y)\left(g^{\prime}(y)\right)^{2}+2=0
$$

with $m=n_{1}+2 n_{2}-1 \geq 3$. This leads to simple explicit examples of harmonic maps having noncontact boundary values: 
Proposition 3.5. Assume that $f: N \rightarrow N$ is a Lie group homomorphism satisfying $f_{1}^{n_{1}+1}=1$ and $f_{j}^{\alpha}=0$ if $j \neq 1$ or $\alpha \neq n_{1}+1$. Define

$$
h(y, n)=\left(\sqrt[4]{\frac{4}{m+1}} y^{\frac{1}{2}}, f(n)\right) .
$$

Then $h: R^{+} \times N \rightarrow R^{+} \times N$ is a harmonic map which assumes the boundary values $f$ continuously.

Proof. In view of the discussion above, one just needs to verify that $g(y)=$ $\sqrt[4]{\frac{4}{m+1}} y^{\frac{1}{2}}$ satisfies (3.4). This is an elementary calculation.

\section{FAMily OF SOLUTiONS}

The harmonic map constructed in Proposition 3.5 is quite special, since it is based on solving the nonlinear differential equation (3.4) in closed form. In this section, we construct an infinite family of harmonic maps, all having the same boundary value $f$, which extend the solution of Proposition 3.5. Our approach is based upon the work of Ecomomakis [3]. He extended the identity map of hyperbolic space to an infinite family of maps, all agreeing with the identity map on the boundary.

The first step is a formal perturbation expansion near $y=0$. We seek an approximate solution to (3.4) given by

$$
g(y) \sim \sqrt[4]{\frac{4}{m+1}}\left(y^{\frac{1}{2}}+a y^{\nu}\right)
$$

for some constants $\nu>\frac{1}{2}$ and $a$. Substituting into (3.4), the highest order term leads to the quadratic equation $\nu^{2}-(m+2) \nu-\left(\frac{6 m+5}{4}\right)=0$. Only one of the roots is positive, so we must take $2 \nu=\left[m+2+\sqrt{(m+2)^{2}+6 m+5}\right]$. Since $m \geq 3$, the required condition $\nu>\frac{1}{2}$ holds. The constant $a$ may be chosen arbitrarily.

With this choice of $\nu$, we try a higher order correction

$$
g(y) \sim \sqrt[4]{\frac{4}{m+1}}\left(y^{\frac{1}{2}}+a y^{\nu}+b y^{s}\right)
$$

for $b$ and $s>\nu$ constants. Calculating the derivatives and retaining the highest order terms in (3.4), leads to $s=2 \nu-\frac{1}{2}$ and

$$
b=-\left(s^{2}-(m+2) s-\frac{6 m+5}{4}\right)^{-1}\left(2 \nu^{2}-5 \nu-3 m \nu-3 m / 2-1\right) a^{2} .
$$

Note that $\nu>\frac{1}{2}$ ensures that $s>\nu$.

Following the technique of [3], we use the contraction mapping principle to establish rigorously that there is a solution of (3.4), with the asymptotic behavior (4.1), defined near $y=0$. Define new dependent variables $\mathrm{v}$ and $w$ by

$$
\begin{aligned}
\frac{g^{\prime}}{g} & =\frac{1}{2} y^{-1}+\mathrm{v}, \\
\mathrm{v} & =c y^{\nu-\frac{3}{2}}+w .
\end{aligned}
$$


Using (4.1), we expect $c=a\left(\nu-\frac{1}{2}\right)$. Also, the function $w$ should vanish to order $2 \nu-2$ at $y=0$. It follows that

$$
g(y)=\sqrt[4]{\frac{4}{m+1}} y^{\frac{1}{2}} \exp \left(\int_{0}^{y} \mathrm{v}(t) d t\right)
$$

The second order nonlinear differential equation (3.4) for $g$ is equivalent to the following integral-differential equation for v:

$$
\frac{d}{d y}\left(y^{-m} \mathrm{v}\right)=\left(\frac{m+1}{2}\right) y^{-m-2}\left(1-\exp \left(-4 \int_{0}^{y} \mathrm{v}(t) d t\right)\right) .
$$

Changing the dependent variable from $\mathrm{v}$ to $w$ gives

$$
\begin{aligned}
\frac{d}{d y}\left(y^{-m} w\right)=( & \left.\frac{m+1}{2}\right) y^{-m-2}\left(1-\exp \left(-4 \int_{0}^{y} w(t) d t-4 c\left(\nu-\frac{1}{2}\right)^{-1} y^{\nu-\frac{1}{2}}\right)\right) \\
& -c\left(\nu-m-\frac{3}{2}\right) y^{\nu-m-\frac{5}{2}}
\end{aligned}
$$

Consider the complete metric space, defined for given positive constants $K$ and $\epsilon$ by

$$
\Lambda_{\epsilon, K}^{2 \nu-2}=\left\{w \in C(0, \epsilon)\left|\sup _{0<y<\epsilon}\right| y^{2-2 \nu} w(y) \mid \leq K\right\}
$$

with

$$
\|w\|_{\epsilon}^{2 \nu-2}=\sup _{0<y<\epsilon}\left|y^{2-2 \nu} w(y)\right|
$$

Our formal calculation leads us to hope that, for any choice of $c$, the equation (4.4) has a solution $w$ belonging to $\Lambda_{\epsilon, K}^{2 \nu-2}$. This should give a family of solutions, parametrized by $c$ or equivalently $a$, to (3.4).

Define

$$
\begin{aligned}
& T w(x) \\
& =x^{m} \int_{0}^{x}\left[\left(\frac{m+1}{2}\right) y^{-m-2}\left(1-\exp \left(-4 \int_{0}^{y} w(t) d t-4 c\left(\nu-\frac{1}{2}\right)^{-1} y^{\nu-\frac{1}{2}}\right)\right)\right. \\
& \left.\quad-c\left(\nu-m-\frac{3}{2}\right) y^{\nu-m-\frac{5}{2}}\right] d y .
\end{aligned}
$$

It suffices to show that $T$ is a contraction mapping on some $\Lambda_{\epsilon, K}^{2 \nu-2}$. Existence of a solution to (4.4) will then follow from the contraction mapping principle.

We first need to establish

Proposition 4.5. Suppose that the parameter $c$ is fixed. For $K(c)$ sufficiently large and $\epsilon=\epsilon(c, K)$ sufficiently small, $T$ maps $\Lambda_{\epsilon, K}^{2 \nu-2}$ into itself.

Proof. Define variables $q$ and $\psi$ by

$$
\begin{aligned}
& q=\exp \left(-4 \int_{0}^{y} w(t) d t\right)-1, \\
& \psi=\exp \left(-4 c\left(\nu-\frac{1}{2}\right)^{-1} y^{\nu-\frac{1}{2}}\right)-1+4 c\left(\nu-\frac{1}{2}\right)^{-1} y^{\nu-\frac{1}{2}} .
\end{aligned}
$$


It is straightforward to estimate the norms of these functions in the space $\Lambda_{\epsilon, K}^{2 \nu-1}$,

$$
\begin{aligned}
\|\psi\|_{\epsilon}^{2 \nu-1} & \leq \frac{64 c^{2}}{(2 \nu-1)^{2}}, \\
\|q\|_{\epsilon}^{2 \nu-1} & \leq \frac{5}{2 \nu-1}\|w\|_{\epsilon}^{2 \nu-2} .
\end{aligned}
$$

Using the quadratic equation $\nu^{2}-(m+2) \nu-(6 m+5) / 4=0$, one verifies that

$$
T w(x)=x^{m} \int_{0}^{x}\left(\frac{m+1}{2}\right) y^{-m-2}\left[q\left(4 c\left(\nu-\frac{1}{2}\right)^{-1} y^{v-\frac{1}{2}}-1\right)-\psi(1+q)\right] d y .
$$

Thus

$$
\begin{aligned}
|T w(x)| \leq & x^{2 v-2}\left(\frac{m+1}{2}\right)\left(1+4|c| \frac{\epsilon^{\nu-\frac{1}{2}}}{\nu-\frac{1}{2}}\right)\left(\frac{1}{2 \nu-m-2}\right)\|q\|_{\epsilon}^{2 \nu-1} \\
& +x^{2 \nu-2}\left(\frac{m+1}{2}\right)\|\psi\|_{\epsilon}^{2 \nu-1} \exp \left(4\|w\|_{\epsilon}^{2 \nu-2} \frac{\epsilon^{2 \nu-1}}{2 v-1}\right)(2 \nu-m-2)^{-1} .
\end{aligned}
$$

If $\|w\|_{\epsilon}^{2 \nu-2} \leq K$, this gives

$$
\begin{aligned}
\|T w\|_{\epsilon}^{2 \nu-2} \leq & \left(\frac{5(m+1) K}{2}\right)(2 \nu-1)^{-1}(2 \nu-m-2)^{-1}\left(1+4|c|\left(\nu-\frac{1}{2}\right)^{-1} \epsilon^{\nu-\frac{1}{2}}\right) \\
& +32(m+1) c^{2}(2 \nu-1)^{-2}(2 \nu-m-2)^{-1} \exp \left(4 K(2 v-1)^{-1} \epsilon^{2 \nu-1}\right) .
\end{aligned}
$$

Since $5(m+1)<2(2 \nu-1)(2 \nu-m-2)$, we may choose $K(c)$ sufficiently large and $\epsilon=\epsilon(c, K)$ sufficiently small to guarantee that $\|T w\|_{\epsilon}^{2 \nu-2} \leq K$. Therefore $T$ maps the space $\Lambda_{\epsilon, K}^{2 \nu-2}$ into itself.

Suppose that $K$ is determined so that the hypothesis of Proposition 4.5 holds. It remains to show that $T$ is a contraction mapping on $\Lambda_{\epsilon, K}^{2 \nu-2}$. One has

$$
\begin{aligned}
& \left|T w_{1}(x)-T w_{2}(x)\right| \\
& \leq x^{m} \int_{0}^{x}\left(\frac{m+1}{2}\right) y^{-m-2} \exp \left(-4 c\left(\nu-\frac{1}{2}\right)^{-1} y^{\nu-\frac{1}{2}}\right) \mid \exp \left(-4 \int_{0}^{y} w_{1}(t)\right) \\
& \quad-\exp \left(-4 \int_{0}^{y} w_{2}(t) d t\right) \mid d y .
\end{aligned}
$$

For sufficiently small $\epsilon$, this gives

$$
\left\|T w_{1}-T w_{2}\right\|_{\epsilon}^{2 \nu-2} \leq 4(m+1)(2 \nu-m-2)^{-1}(2 \nu-1)^{-1}\left\|w_{1}-w_{2}\right\|_{\epsilon}^{2 \nu-2} .
$$

Since $4(m+1)<(2 \nu-m-2)(2 \nu-1)$, we have a contraction mapping $T: \Lambda_{\epsilon}^{2 \nu-2} \rightarrow$ $\Lambda_{\epsilon}^{2 \nu-2}$.

The results proved above give a family of solutions $w_{c}$ to the integral-differential equation (4.4). Taking

$$
g_{a}(y)=\sqrt[4]{\frac{4}{m+1}} y^{\frac{1}{2}} \exp \left(\int_{0}^{y}\left[w_{c}(t)+c t^{\nu-\frac{3}{2}}\right] d t\right)
$$

where $c=a\left(\nu-\frac{1}{2}\right)$, gives a family of solutions to the second order nonlinear differential equation (3.4). Also, one has the asymptotic relation (4.1), which determines $a$. So far, these solutions are only determined locally, near $y=0$. 
To extend the domain of definition of $g_{a}(y)$ to all $y \geq 0$, we assume that $a$ is positive. Then $v_{c}=c y^{\nu-\frac{3}{2}}+w_{c}$ is positive for sufficiently small $y>0$. Let $S$ be the supremum of all $s>0$, so that $\mathrm{v}_{c}$ extends to a strictly positive solution of (4.3), on the interval $(0, s)$. By uniqueness for the ordinary differential equation (3.4), the extension $\mathrm{v}_{c}$ is unique.

If $S$ is finite, then on the interval $(0, S)$ we have

$$
0 \leq \frac{d}{d y}\left(y^{-m_{\mathrm{v}}}\right) \leq\left(\frac{m+1}{2}\right) y^{-m-2}
$$

Since $y^{-m} \mathrm{v}$ is increasing and bounded above on $(0, S)$, we have existence of a finite limit $\lim _{y \rightarrow S} \mathrm{v}(y)>0$. Using the integral formula (4.2), we deduce the existence of the limits $\lim _{y \rightarrow S} g(y)$ and $\lim _{y \rightarrow S} g^{\prime}(y)$. Local existence theory for the equation (3.4) provides an extension of the solution $g$ to $(0, S+\delta)$, for some $\delta>0$. Consequently, the solution v of (4.3) extends to $(0, S+\delta)$. It follows that $S$ is infinite.

The resulting family of harmonic maps is described in the following extension of Proposition 3.5:

Proposition 4.6. Assume that $f: N \rightarrow N$ is a Lie group homomorphism satisfying $f_{1}^{n_{1}+1}=1$ and $f_{j}^{\alpha}=0$ for $j \neq 1$ or $\alpha \neq n_{1}+1$. Define, for $a>0$,

$$
h_{a}(y, n)=\left(g_{a}(y), f(n)\right)
$$

Then $h_{a}: R^{+} \times N \rightarrow R^{+} \times N$ is a family of harmonic maps, all of which assume the same boundary value $f$, continuously in $(y, n)$. The parameter a may be determined from the asymptotic behavior of $g_{a}$, as y approaches zero,

$$
g_{a}(y) \sim \sqrt[4]{\frac{4}{m+1}}\left(y^{\frac{1}{2}}+a y^{\nu}\right), \quad \nu>\frac{1}{2}
$$

\section{Existence of global SOlutions}

The purpose of the present section is to show the existence of harmonic self maps $h$ of rank one symmetric spaces $M$, which extend continuously to the closure $\bar{M}$, and have noncontact boundary values $f$. The explicit solution of Proposition 3.5, and the family of solutions constructed in Proposition 4.6, only extend continuously to $\bar{M}-\infty=[0, \infty) \times N$. In fact, their boundary values $f: N \rightarrow N$ do not extend continuously to $N \cup\{\infty\} \rightarrow N \cup\{\infty\}$. The point is that in the examples considered above, the map $f$ is a Lie group homomorphism, but not an automorphism.

Lie group automorphisms of $N$ are in one to one correspondence with automorphisms of the Lie algebra $\mathfrak{n}$. For any Lie group, an automorphism $f$ of the Lie group induces an automorphism $f_{*}$ of the corresponding Lie algebra. Since $N$ is simply connected and nilpotent, $\exp : \mathfrak{n} \rightarrow N$ is a diffeomorphism. Given $f_{*}: \mathfrak{n} \rightarrow \mathfrak{n}$, one can define $f$ by $f(\exp v)=\exp \left(f_{*} v\right)$. It follows from the Campbell-Hausdorff formula that $f: N \rightarrow N$ is a Lie group automorphism.

The differential $f_{*}$ of an automorphism $f: N \rightarrow N$ must preserve the center $\mathfrak{n}_{2}$ of the Lie algebra $\mathfrak{n}=\mathfrak{n}_{1} \oplus \mathfrak{n}_{2}$. Thus the components $f_{j}^{\beta}=0$, for $1 \leq \beta \leq n_{1}$ and $n_{1}+1 \leq j \leq n_{1}+n_{2}$. The required condition (3.2) reduces to

$$
\sum_{j=1}^{n_{1}} \sum_{\beta=1}^{n_{1}} \sum_{\gamma=n_{1}+1}^{n_{1}+n_{2}} a_{\alpha \beta}^{\gamma-n_{1}} f_{j}^{\beta} f_{j}^{\gamma}=0
$$

for $1 \leq \alpha \leq n_{1}$. 
To show that there are examples of noncontact Lie algebra automorphisms satisfying (5.1), assume that $f_{j}^{\beta}=\delta_{j \beta}$, for $1 \leq j, \beta \leq n_{1}$. Then (5.1) becomes

$$
\sum_{\beta=1}^{n_{1}} \sum_{\gamma=n_{1}+1}^{n_{1}+n_{2}} a_{\alpha \beta}^{\gamma-n_{1}} f_{\beta}^{\gamma}=0 .
$$

This represents $n_{1}$ linear equations, parametrized by $1 \leq \alpha \leq n_{1}$, in the $n_{1} n_{2}$ unknowns $f_{\beta}^{\gamma}$. Since the system is homogeneous, there exists a nontrivial solution provided $n_{2}>1$. The corresponding Lie algebra automorphism is given by $f_{*}\left(X_{i}\right)=$ $X_{i}+\sum_{\gamma} f_{i}^{\gamma} Z_{\gamma-n_{1}}$ and $f_{*}\left(Z_{i}\right)=Z_{i}$. Since the $Z_{i}$ are central, $f_{*}$ clearly preserves the Lie bracket.

For a general automorphism $f$, the $n_{1} \times n_{1}$ matrix $f_{j}^{\beta}, 1 \leq j, \beta \leq n_{1}$, must be invertible. If $n_{2}=1$, then for each $1 \leq \alpha \leq n_{1}$, there is a unique $1 \leq \beta \leq n_{1}$, with $a_{\alpha \beta}^{\gamma-n_{1}} \neq 0$, in the standard basis for the Lie algebra [5]. Thus (5.1) reduces to

$$
\sum_{j=1}^{n_{1}} f_{j}^{\beta} f_{j}^{\gamma}=0, \quad 1 \leq \beta \leq n_{1} .
$$

So $f_{j}^{\gamma}=0$ and $f_{*}$ can only be the identity map. Therefore, one must have $n_{2}>1$ in order to find noncontact automorphisms satisfying (5.1).

Suppose that $f: N \rightarrow N$ is a Lie group automorphism whose differential satisfies (5.1). We extend $f$ to $h: R^{+} \times N \rightarrow R^{+} \times N$ by $h(y, n)=(g(y), f(n))$. The requirement that $h$ is harmonic reduces to the ordinary differential equation in $g$, given by (3.3). We note that (3.3) is of the form

$$
\begin{aligned}
y^{2} g^{\prime \prime}(y)-m y g^{\prime}(y)-y^{2}\left(g^{\prime}(y)\right)^{2}(g(y))^{-1}+c_{1} y^{2}( & g(y))^{-1} \\
& +2\left[c_{3} y^{2}+c_{4} y^{4}\right](g(y))^{-3}=0 .
\end{aligned}
$$

Here $c_{2}=\sum_{j=n_{1}+1}^{n_{1}+n_{2}} \sum_{\gamma=1}^{n_{1}} f_{j}^{\gamma} f_{j}^{\gamma}=0$, since $f_{*}$ must preserve the center of $\mathfrak{n}$. All of the other $c_{i}$ are positive constants. We require $c_{3}$ to be positive so that $f$ is noncontact.

One may conveniently change the independent variable in our differential equation. Suppose that

$$
g(y)=\sqrt[4]{\frac{4 c_{3}}{m+1}} y^{\frac{1}{2}} w(y)=b y^{\frac{1}{2}} w(y)
$$

Then

$$
y^{2} w^{3} w^{\prime \prime}-m y w^{3} w^{\prime}-y^{2} w^{2}\left(w^{\prime}\right)^{2}+\left(\frac{m+1}{2}\right)\left(1-w^{4}\right)+a_{1} y w^{2}+a_{4} y^{2}=0 .
$$

Here $a_{1}=c_{1} / b^{2}, a_{4}=2 c_{4} / b^{4}$, with $b=\sqrt[4]{\frac{4 c_{3}}{m+1}}$. Clearly, $a_{1}$ and $a_{4}$ are positive. Also, since $c_{3}=\sum_{j=1}^{n_{1}} \sum_{\gamma=n_{1}+1}^{n_{1}+n_{2}} f_{j}^{\gamma} f_{j}^{\gamma}$ and (5.2) is homogeneous, there exist noncontact automorphisms $f$ so that $a_{1}$ and $a_{4}$ are arbitrarily small.

If $w_{1}(y)=1$, then one has

$$
\begin{aligned}
& y^{2} w_{1}^{3} w_{1}^{\prime \prime}-m y w_{1}^{3} w_{1}^{\prime}-y^{2} w_{1}^{2}\left(w_{1}^{\prime}\right)^{2}+\left(\frac{m+1}{2}\right)\left(1-w_{1}^{4}\right)+a_{1} y w_{1}^{2}+a_{4} y^{2} \\
& =a_{1} y+a_{4} y^{2}>0 .
\end{aligned}
$$


Conversely, for $w_{2}(y)=e^{y}$,

$$
\begin{gathered}
y^{2} w_{2}^{3} w_{2}^{\prime \prime}-m y w_{2}^{3} w_{2}^{\prime}-y^{2} w_{2}^{2}\left(w_{2}^{\prime}\right)^{2}+\left(\frac{m+1}{2}\right)\left(1-w_{2}^{4}\right)+a_{1} y w_{2}^{2}+a_{4} y^{2} \\
=-m y e^{4 y}+\left(\frac{m+1}{2}\right)\left(1-e^{4 y}\right)+a_{1} y e^{2 y}+a_{4} y^{2}<0
\end{gathered}
$$

provided $a_{1}$ and $a_{4}$ are sufficiently small.

This suggests that (5.3) should have a solution $w(y)$ satisfying $1 \leq w(y) \leq e^{y}$. For such a $w(y)$, the corresponding $g(y)$ satisfies $b y^{\frac{1}{2}} \leq g(y) \leq b y^{\frac{1}{2}} e^{y}$. So $h$ : $R^{+} \times N \rightarrow R^{+} \times N$ will be a harmonic map, extending continuously to $\bar{M}$ with noncontact boundary values $f$.

We will apply Theorem 7.1 of [1] to show the existence of $w(y)$. Some care must be taken since (5.3) is not uniformly Lipschitz in $w^{\prime}(y)$. Define, for small $\epsilon>0$,

$$
G(p)= \begin{cases}p^{2}, & |p| \leq \exp \left(\epsilon^{-1}\right), \\ \exp \left(\epsilon^{-1}\right)|p|, & |p| \geq \exp \left(\epsilon^{-1}\right) .\end{cases}
$$

Consider the following modification of (5.3),

$$
y^{2} w^{3} w^{\prime \prime}-m y w^{3} w^{\prime}-y^{2} w^{2} G\left(w^{\prime}\right)+\left(\frac{m+1}{2}\right)\left(1-w^{4}\right)+a_{1} y w^{2}+a_{4} y^{2}=0 .
$$

Assume that $a_{1}$ and $a_{4}$ are sufficiently small. Then (5.4) satisfies the hypotheses of Theorem 7.1 in [1], with subsolution $w_{1}(y)=y$ and supersolution $w_{2}(y)=e^{y}$, on the interval $\left(\epsilon, \epsilon^{-1}\right)$. We deduce that (5.4) has a solution $w(y)$, for $\epsilon<y<\epsilon^{-1}$, satisfying the bounds $w_{1}(y) \leq w(y) \leq w_{2}(y)$.

To obtain a solution of $(5.3)$, we need to establish an a priori bound for $\left|w^{\prime}(y)\right|$. If $a>0$, let

$$
C_{1}(a)=\sup _{\substack{a / 2 \leq y \leq a+1 \\ w_{1}(y) \leq \mathrm{v} \leq w_{2}(y)}}\left|\left(\frac{m+1}{2}\right)\left(1-\mathrm{v}^{4}\right)+a_{1} y \mathrm{v}^{2}+a_{4} y^{2}\right| .
$$

Clearly, for $\epsilon(a)$ sufficiently small, the solution $w(y)$, of (5.4), satisfies $y^{2} w^{3} w^{\prime \prime} \geq$ $y w^{2}\left(m w w^{\prime}+y G\left(w^{\prime}\right)\right)-C_{1}(a)$, for $y \in[a / 2, a+1]$. Choose $D_{1}(a)$ so that $\left|w^{\prime}(y)\right| \geq$ $D_{1}(a)$ implies $w^{\prime \prime}(y)>0$, for each $y \in[a / 2, a+1]$. If $w^{\prime}(a) \geq D_{1}(a)$, then $w^{\prime}(y) \geq$ $D_{1}(a)$, for $y \in[a, a+1]$. If $w^{\prime}(a) \leq-D_{1}(a)$, then $w^{\prime}(y) \leq-D_{1}(a)$, for $y \in[a / 2, a]$. This is because the interval where the bound on $w^{\prime}(y)$ holds is both open and closed.

If $w^{\prime}(a) \geq D_{1}(a)$, we use the fundamental theorem of calculus to deduce that $w(a+1) \geq D_{1}(a)+1$. Since $D_{1}(a)$ was only required to be sufficiently large, $D_{1}(a)$ may be replaced by $D_{2}(a)=\max \left(D_{1}(a), e^{a+2}\right)$. Then one has a contradiction to the bound $w(a+1) \leq w_{2}(a+1)$. Similarly, if $w^{\prime}(a) \leq-D_{1}(a)$, then $w(a / 2) \geq$ $1+a D_{1}(a) / 2$. Replacing $D_{1}(a)$ by $D_{2}(a)=\max \left(D_{1}(a), 2 e^{a} / a\right)$ gives a contradiction to $w(a / 2) \leq w_{2}(a / 2)$.

Thus, the solution to (5.4), guaranteed by Theorem 7.1 of [1], satisfies $\left|w^{\prime}(a)\right| \leq$ $D_{2}(a)$ and $w_{1}(a) \leq w(a) \leq w_{2}(a)$. Letting $\epsilon \rightarrow 0$ and applying the Arzela-Ascoli theorem leads to a solution $w(y)$ of (5.3) satisfying $w_{1}(y) \leq w(y) \leq w_{2}(y)$, for all $0<y<\infty$.

We summarize the main conclusion of this section as

Proposition 5.5. Let $M$ be a rank one symmetric space, with $n_{2}>1$. Then there exist harmonic maps $h: M \rightarrow M$, which extend continuously to the compactification $\bar{M}$, and assume noncontact boundary values $f$. 
The solutions, whose existence has just been established, are defined and continuous on all of $\bar{M}$. In previous sections, the harmonic maps found were only continuous on $\bar{M}-\infty$. However, the earlier methods were more constructive. In particular, the harmonic map of Proposition 3.5 is given quite explicitly.

\section{REFERENCES}

1. P. Bailey, L. Shampine, and P. Waltman, Nonlinear two point boundary value problems, Academic Press, NY and London, 1968. MR 37:6524

2. H. Donnelly, Dirichlet problem at infinity for harmonic maps: Rank one symmetric spaces, Transactions of the American Mathematical Society 344 (1994), 713-735. MR 95c:58045

3. M. Economakis, A counterexample to uniqueness and regularity for harmonic maps between hyperbolic spaces, Journal of geometric analysis 3 (1993), 27-36. MR 93m:58024

4. P. Li and L. F. Tam, Uniqueness and regularity of proper harmonic maps, Ann. Math. 137 (1993), 167-201. MR 93m:58027

5. G. Mostow, Strong rigidity of locally symmetric spaces, Annals of Mathematics Studies, 78, Princeton University Press, (1973). MR 52:5784

6. M. Wolf, Infinite energy harmonic maps and degeneration of hyperbolic surfaces in moduli space, J. Diff. Geom. 33 (1991), 487-539. MR 92b:58055

Department of Mathematics, Purdue University, West Lafayette, Indiana 47907 